\title{
FUNCIONALIDADE ECOLÓGICA DE SISTEMAS AGROFLORESTAIS BIODIVERSOS: USO DA SERAPILHEIRA COMO INDICADOR DA RECUPERAÇÃO DE ÁREAS DE PRESERVAÇÃO PERMANENTE
}

\author{
Márcia Cristina Soares de Souza ${ }^{1}$, Fatima Conceição Márquez Piña-Rodrigues ${ }^{2}$, José Carlos Casagrande ${ }^{3}$, \\ Simone Ferreira da Silva ${ }^{4}$, Rafael Nogueira Scoriza ${ }^{5}$ \\ ${ }^{1}$ Centro Apta Citros Sylvio, Técnica de Apoio à Pesquisa Científica e Tecnológica, Cordeiropolis, São Paulo, Brasil - \\ mcssouza@yahoo.com.br \\ ${ }^{2}$ Universidade Federal de São Carlos, Departamento de Ciências Ambientais, Sorocaba, São Paulo, Brasil - fpina @ufscar.br \\ ${ }^{3}$ Universidade Federal de São Carlos, Departamento de Recursos Naturais e Proteção Ambiental, Araras, São Paulo, Brasil - \\ bighouse@ccc.ufscar.br \\ ${ }^{4}$ São Paulo, SP, Brasil, sifsil@yahoo.com.br \\ ${ }_{5}^{5}$ Embrapa Agrobiologia, Seropédica, Rio de Janeiro, Brasil - rafaelscoriza@ gmail.com
}

Recebido para publicação: 13/01/2014 - Aceito para publicação: 28/11/2015

\begin{abstract}
Resumo
As recentes alterações na legislação brasileira incentivam a utilização de sistemas produtivos em áreas de preservação. Para avaliar a funcionalidade ecológica quanto ao aporte de biomassa e nutrientes, foi feito estudo de uma área de floresta e de quatro modelos de sistemas agroflorestais (SAFs) biodiversos e multiestratificados, com plantio de uma (solteira) a duas (casada) mudas por cova e com e sem consórcio de leguminosas, em Áreas de Preservação Permanente (APPs) no município de Paraty, RJ. A produção e o aporte de nutrientes foram maiores na

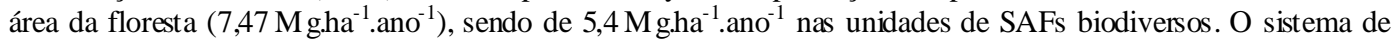
plantio e o uso de leguminosas não promoveram incrementos no aporte de biomassa, sendo influenciados pelo manejo, que também afetou a sazonalidade da dep osição. $\mathrm{O}$ aporte de serapilheira foi um eficiente indicador, sendo sensível para detectar alterações temporais e de manejo nas áreas dos sistemas agroflorestais estudados.

Palavras-chave: Mata ciliar; indicadores de sustentabilidade; políticas públicas.
\end{abstract}

\begin{abstract}
Ecological functionality biodiverse agroforestry systems: use of litterfall as an indicator of the recovery of permanent preservation areas. A recent change in legislation encourages the use of production systems in conservation areas. To assess the ecological functionality of biomass and nutrients deposition, four models of agroforestry system (AFS) biodiverse and multi-layered with planting a (single) to two (married) seedlings per hole, with and without legumes consortium were studied and compared to a forest in Areas of Permanent Preservation (APPs) in the municipality of Paraty, RJ. The production and supply of nutrients was higher in forest area $\left(7.47 \mathrm{Mg}^{-1} \mathrm{~h}^{-1} \cdot \mathrm{yr}^{-1}\right)$, than in AFS biodiverse $\left(5.4 \mathrm{Mg}^{-h^{-1}} \cdot \mathrm{yr}^{-1}\right)$. The plantation sy stem and the use of legumes did not promote an increase in the biomass input, being influenced by management, which also affected the seasonality of deposition. The contribution of litter was an effective indicator, being sensitive to detect temporal and management in the areas of agroforestry systems studied changes.

Keywords: Agroforestry; gallery forest; sustainability indicators; public policies.
\end{abstract}

\section{INTRODUÇÃO}

A restauração de áreas degradadas é um processo que evoluiu em suas bases conceituais, desde a implantação de um sistema de plantio monoespecífico até a busca de alta diversidade de espécies e o restabelecimento de processos ecológicos (OLIVEIRA; ENGEL, 2011). Atualmente, a restauração tem como paradigma o foco na interação entre a biodiversidade e a funcionalidade ecológica do ecossistema, baseada no conceito Biodiversity-Ecosystem Functioning (BEF) (NAEEM, 2006). Nessa perspectiva, a comunidade e o ecossistema são inseparáveis, e a biodiversidade é o fator-chave para regular o funcionamento do ecossistema, especialmente pelo seu importante papel de resiliência. Os sistemas agroflorestais (SAFs) formados pela combinação de espécies arbóreas com culturas agrícolas têm sido também utilizados no Brasil como estratégia na recuperação de ecossis temas ameaçados (VIVAN; FLORIANI, 2006). Es ses sis temas têm como objetivo restaurar as funções ambientais e aumentar a biodiversidade (SILVEIRA et al., 2007) e a produtividade agrícola e florestal.

Dentre os vários sistemas agroflorestais existentes, o Sistema Agroflorestal Regenerativo e Análogo (SAFRA) caracteriza-se por aliar a alta diversidade de espécies com práticas de manejo que visam reproduzir os

FLOREST A, Curitiba, PR, v. 46, n. 1, p. 75 - 82, jan. / mar. 2016.

Souza, M. C. S. de et al.

ISSN eletrônico 1982-4688

DOI: $10.5380 /$ rf.v46il .34991 
processos sucessionais ocorrentes em florestas naturais (CAMPELLO et al., 2007). Por ser um sistema mu ltiestratificado e diverso (SILVEIRA et al., 2007), espera-se que contribua de forma eficiente na proteção e conservação de áreas que necessitam de recuperação.

A Resolução do Conselho Nacional do Meio A mbiente (CONAMA) nº 429, de 28 de fevereiro de 2011, estabeleceu a possibilidade do plantio consorciado de espécies nativas perenes frutíferas e outros produtos vegetais em APPs para a extração sustentável não madeireira (CONAMA, 2011). Porém, apesar desses mecanismos legais, ainda é fundamental viabilizar a recuperação em pequenas propriedades rurais, com metodologias que conciliem o processo de restauração ambiental e a produção sustentável. Por is so, o período recomendado pelos mecanis mos legais para a manutenção dos sistemas agroflorestais em áreas de preservação permanente pode não ser suficiente para se obter produção e nem a restauração de sua funcionalidade ecológica.

Como ressaltam Ramos Filho et al. (2007), o sucesso dessa atividade como restauradora das funções ecológicas e de produção requer estudos sistemáticos por meio de indicadores, que forneçam informações sobre a evolução do processo de restauração. Entre os potenciais indicadores, a serapilheira forma uma parte substancial na cadeia trófica do solo. Quando depositada sobre o solo, adquire importância na melhoria e manutenção da fertilidade, na atividade biológica e no estabelecimento de um microclima favorável, o que influencia diretamente a biomassa microbiana e a comunidade da macrofauna (CUNHA NETO et al., 2013; ASHFORD et al., 2013). Esse potencial indicador reflete diversos atributos físicos e biológicos, determinantes para a deposição da serapilheira nas diversas tipologias florestais (GOMES et al., 2010), que pode ser uma ferramenta importante para a avaliação da recuperação do ecossistema.

Considerando o exposto e a importância de se estabelecer sistemas produtivos de recuperação em APPs, o presente trabalho teve como objetivo avaliar: (a) a eficiência do modelo multiestratificado de sistemas agroflorestais regenerativos e análogos na restauração dos processos ecológicos de aporte de biomassa e de nutrientes; (b) as práticas de manejo na produtividade e na sazonalidade do aporte de biomassa; e (c) o aporte de serapilheira co mo indicador da funcionalidade ecológica dos sistemas agroflorestais.

\section{MATERIAL E MÉTODOS}

\section{Área de estudo}

O trabalho foi realizado em APPs recuperadas com sistemas agroflorestais, localizadas nas

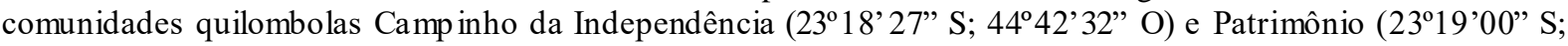
$44^{\circ} 43^{\prime} 23^{\prime \prime}$ O) e em área de floresta secundária contígua, utilizada como referência. Essas unidades estão situadas na área de proteção ambiental (APA) do Cairuçu e no entorno do Parque Nacional da Serra da Bocaina, no distrito de Paraty-Mirim, município de Paraty, RJ, Brasil. O clima da região é do tipo CWa, com precipitação anual média de $1.946 \mathrm{~mm}$ e temperatura média anual de $19,5{ }^{\circ} \mathrm{C}$ (ALVARES et al., 2013). A cobertura vegetal original da área é do tipo Ombrófila Densa Submontana, segundo o Instituto Brasileiro de Geografia e Estatística (IBGE, 2012), c lassificada como em estágio sucessional médio de regeneração segundo a Resolução CONAMA 01 (CONAMA, 1994).

\section{Caracterização do sistema agrofl orestal}

As unidades experimentais de sistemas agroflorestais foram implantadas em março de 2003 baseadas no modelo de Sistemas Agroflorestais Regenerativos e Análogos (SAFRA). Cada unidade experimental de $400 \mathrm{~m}^{2}$ foi constituída por um dos tratamentos $(n=4)$, consorciando espécies leguminosas e não leguminosas em sistemas de plantio "solteiro" (uma muda por cova) e "casado" (duas mudas por cova) (Tabela 1). As mudas "casadas" no T2 foram guapuruvu (Schizolobium parahyba (Vell.) Blake) x ingá (Inga sp.), araribá (Centrolobium tomentosum Guill. ex Benth) x embira-de-sapo (Lonchocarpus guillemineanus (Tul.) Malme), jatobá (Hymenaea courbaril L.) x banana (Musa sp.) e copaíba (Copaifera langsdorffii Desf.) x banana. No T4, cedro (Cedrela fissilis Vell.) x pau-viola (Citharexilum myriantum Cham.), canela (Nectandra lanceolata Nees.) $\mathrm{x}$ urucum (Bixa orellana L.), jequitibá (Cariniana legalis (Mart.) Kuntze) x banana e bicuíba (Virola bicuhyba (Schott ex Spreng.) Warb.) x banana. No período de 2003 a 2006, foram plantadas espécies agrícolas, como feijão-preto (Phaseolus vulgaris L.), milho (Zea mays L.) e mandioca (Manihot esculenta L.), e de adubação verde, como feijão-de-porco (Canavalia ensiformis (L.) DC.), feijão-guandu (Cajanus cajans (L.) Millsp.) e crotalária (Crotalaria juncea L.), nas entrelinhas das espécies arbóreas.

Nas unidades experimentais do Campinho da Independência, foram realizadas capinas e manutenção da vegetação apenas nos primeiros anos (2003 e 2004). Posteriormente, mes mo sem o fechamento da vegetação, essas atividades deixaram de ser realizadas, havendo apenas a incorporação das leguminosas ao solo (ano 1) e a coleta periódica de produtos. Nas unidades do Patrimônio foi realizada coleta periódica de produtos e práticas 
culturais, como a poda das espécies renovadoras da fertilidade e capina de manutenção até o segundo ano (2005), quando a necessidade dessa operação foi reduzida.

Tabela 1. Composição florística, sistemas de plantio e funções das espécies empregadas nos sistemas agroflorestais, Paraty, RJ.

Table 1. Floristic composition, systems of plantation and functions of species in agrofore stry systems, Paraty, RJ. Single - a seedling/hole; Married - two seedlings/hole.

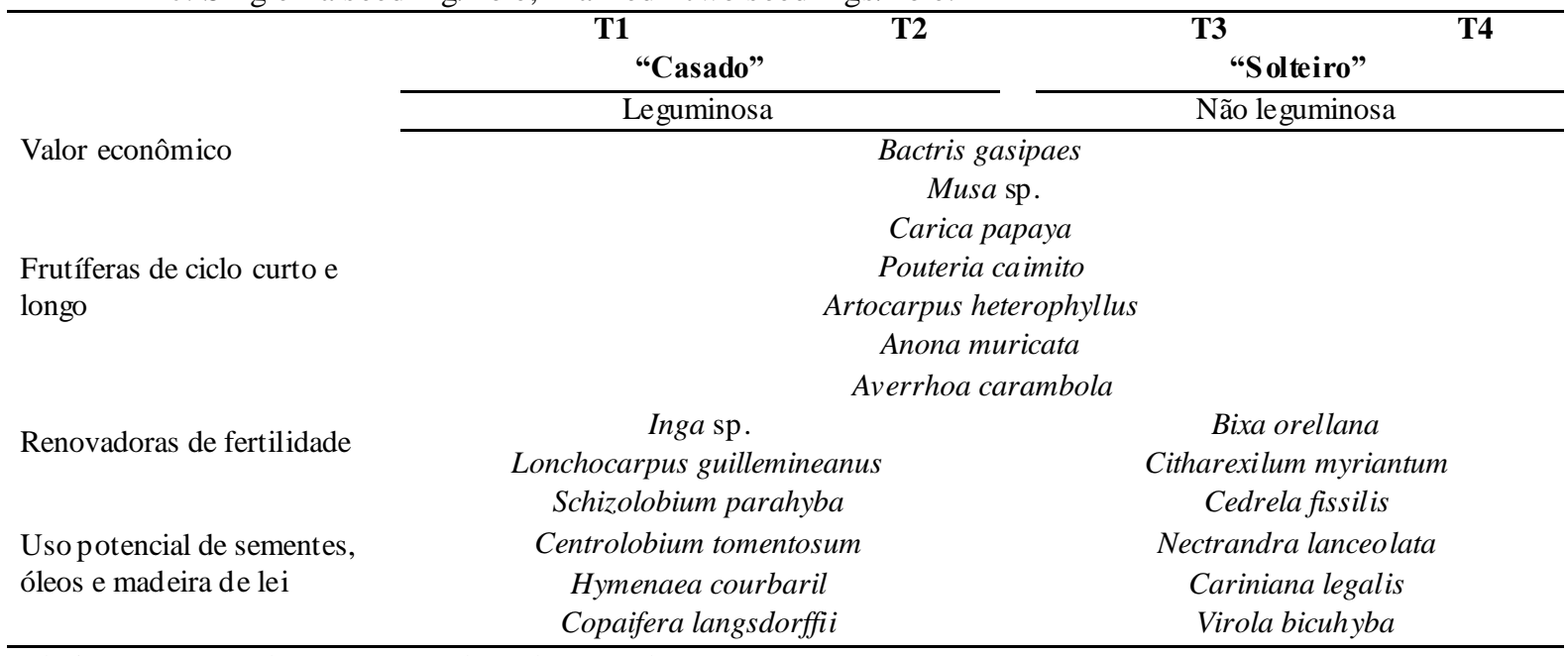

Solteiro - u ma muda/cova; Casado - duas mudas/cova.

\section{Amostragem e produção de serapilheira}

Para estimar a produção de serapilheira, foram instalados oito coletores cônicos de tecido (tipo helanca), com $0,25 \mathrm{~m}^{2}$ de área, dispostos em diagonal, a 1,30 $\mathrm{m}$ do solo, em cada tratamento das unidades experimentais de sistemas agroflorestais e em uma área de floresta secundária utilizada como referência. Mensalmente, no período de outubro de 2007 a setembro de 2008, o material retido nos coletores foi recolhido e separado nas frações folhas, ramos (diâmetro menor que $2 \mathrm{~cm}$ ), partes reprodutivas (flores, frutos e sementes) e resíduos. O material foi seco a $65^{\circ} \mathrm{C}$ por $24 \mathrm{~h}$ e pesado. Para a fração partes reprodutivas, a massa seca em estufa não foi obtida, para evitar possíveis descaracterizações dos padrões morfológicos das sementes para estudos futuros. Para a análise de aporte de nutrientes, o material triado foi homogeneizado, obtendo-se uma amostra por estação (primavera, verão, outono e inverno) em cada um dos cinco tratamentos, e encaminhado para a análise química de nitrogênio $(\mathrm{N})$, fósforo $(\mathrm{P})$, potássio $(\mathrm{K})$, cálcio $(\mathrm{Ca})$ e magnésio $(\mathrm{Mg})$.

\section{Análise dos dados}

Os aportes mensais $\left(\mathrm{Mg} \cdot \mathrm{ha}^{-1}\right)$ e anual $\left(\mathrm{Mg} \cdot \mathrm{ha}^{-1} \cdot \mathrm{ano}^{-1}\right)$ de serapilheira foram realizados a partir da transformação da massa seca de cada fração em relação à área do coletor. Para avaliar as diferenças de produção de serapilheira e aporte de nutrientes entre as áreas e os meses de coleta, foi empregado o modelo geral linear (GLM) balanceado, visando à realização da análise de variância envolvendo variáveis múlt iplas e dependentes. As três áreas e os meses foram avaliados como fatores fixos, e as frações como variáveis dependentes, sendo os coletores as repetições. Para a comparação das médias, foi aplicado o teste de Tukey a 5\% de probabilidade, e para a homogeneidade de variância, o método de Levene, com uso do programa SPSS 13.0.

\section{RES ULTADOS E DISCUSSÃO}

A produção de serapilheira total foi significativamente diferente entre as três áreas de estudo $(\mathrm{F}=22,52$; $p<0,01)$, com maior deposição na área de floresta secundária $\left(7,47 \pm 0,19 \mathrm{Mg}^{-1} \mathrm{a}^{-1} \cdot\right.$ ano $\left.^{-1}\right)$ do que nos SAFs $\left(2,69 \pm 0,79 \mathrm{Mg} \cdot \mathrm{ha}^{-1} \cdot \mathrm{ano}^{-1}\right)$. Do total de biomassa aportado, a fração foliar foi dominante em todas as áreas de estudo (Tabela 2).

Resultado similar foi também obtido por Corrêa et al. (2006), que observou maior produção de serapilheira na vegetação natural (capoeira) do que nos SAFs multiestratificados estudados. Contudo, embora a deposição nos SAFs tenha sido inferior à da floresta estudada, ela se situou na faixa reportada para frag mentos em diferentes estádios sucessionais de Floresta Atlântica, entre 2,1 e 6,9 Mg.ha ${ }^{-1}$.ano ${ }^{-1}$ (ESPIG et al., 2009; NASCIMENTO et al., 2013). O percentual de folhas observado é semelhante ao obtido em outras pesquisas

FLOREST A, Curitiba, PR, v. 46, n. 1, p. 75 - 82, jan. / mar. 2016. 
conduzidas emflorestas tropicais.

Tabela 2. Estimativa do aporte anual de serapilheira $\left(\mathrm{Mg} \cdot \mathrm{ha}^{-1} \cdot \mathrm{ano}^{-1}\right)$ nas unidades experimentais de sistemas agroflorestais e na área de floresta secundária, em Paraty, RJ.

Table 2. Estimated annual deposition of litter $\left(\mathrm{Mg}_{\mathrm{ha}} \mathrm{h}^{-1} \cdot \mathrm{yr}^{-1}\right)$ in experimental areas of agroforestry, and in a secondary forest, Paraty, RJ.

\begin{tabular}{|c|c|c|c|c|c|c|c|c|c|}
\hline \multirow{3}{*}{$\begin{array}{l}\text { Frações da } \\
\text { serapilheira }\end{array}$} & \multicolumn{4}{|c|}{ Campinho da Independência } & \multicolumn{4}{|c|}{ Patrimônio } & \multirow{2}{*}{$\begin{array}{l}\text { Floresta } \\
\text { secundária }\end{array}$} \\
\hline & T1 & T2 & T3 & T4 & T1 & $\mathbf{T 2}$ & T3 & T4 & \\
\hline & \multicolumn{9}{|c|}{ - } \\
\hline Folhas & $\begin{array}{l}2,28 \mathrm{c} \\
(77 \%)\end{array}$ & $\begin{array}{l}2,12 \mathrm{c} \\
(77 \%)\end{array}$ & $\begin{array}{l}1,21 \mathrm{c} \\
(88 \%)\end{array}$ & $\begin{array}{l}0,64 \mathrm{c} \\
(82 \%)\end{array}$ & $\begin{array}{l}2,30 \mathrm{~b} \\
(72 \%)\end{array}$ & $\begin{array}{l}2,32 \mathrm{~b} \\
(78 \%)\end{array}$ & $\begin{array}{l}3,12 \mathrm{~b} \\
(77 \%)\end{array}$ & $\begin{array}{l}2,70 \mathrm{~b} \\
(77 \%)\end{array}$ & $\begin{array}{l}4,64 \mathrm{a} \\
(62 \%)\end{array}$ \\
\hline Ramos & $\begin{array}{c}0,20 \mathrm{~b} \\
(7 \%)\end{array}$ & $\begin{array}{c}0,42 \mathrm{~b} \\
(15 \%)\end{array}$ & $\begin{array}{c}0,02 \mathrm{~b} \\
(2 \%)\end{array}$ & $\begin{array}{c}0,05 \mathrm{~b} \\
(6 \%)\end{array}$ & $\begin{array}{c}0,17 \mathrm{~b} \\
(5 \%)\end{array}$ & $\begin{array}{l}0,32 \mathrm{~b} \\
(11 \%)\end{array}$ & $\begin{array}{c}0,31 \mathrm{~b} \\
(8 \%)\end{array}$ & $\begin{array}{c}0,31 \mathrm{~b} \\
(9 \%)\end{array}$ & $\begin{array}{l}1,50 \mathrm{a} \\
(20 \%)\end{array}$ \\
\hline Partes reprodutivas & $\begin{array}{c}0,13 \mathrm{c} \\
(4 \%)\end{array}$ & $\begin{array}{c}0,09 \mathrm{c} \\
(3 \%)\end{array}$ & $\begin{array}{c}0,05 \mathrm{c} \\
(4 \%)\end{array}$ & $\begin{array}{c}0,03 \mathrm{c} \\
(4 \%)\end{array}$ & $\begin{array}{c}0,52 \mathrm{ab} \\
(16 \%)\end{array}$ & $\begin{array}{c}0,13 \mathrm{~b} \\
(4 \%)\end{array}$ & $\begin{array}{c}0,18 \mathrm{~b} \\
(4 \%)\end{array}$ & $\begin{array}{c}0,31 \mathrm{~b} \\
(9 \%)\end{array}$ & $\begin{array}{c}0,56 \mathrm{a} \\
(7 \%)\end{array}$ \\
\hline Resíduos & $\begin{array}{l}0,35 \mathrm{~b} \\
(12 \%)\end{array}$ & $\begin{array}{c}0,13 \mathrm{~b} \\
(5 \%)\end{array}$ & $\begin{array}{c}0,09 \mathrm{~b} \\
(6 \%)\end{array}$ & $\begin{array}{c}0,07 \mathrm{~b} \\
(9 \%)\end{array}$ & $\begin{array}{c}0,19 \mathrm{~b} \\
(6 \%)\end{array}$ & $\begin{array}{c}0,19 \mathrm{~b} \\
(7 \%)\end{array}$ & $\begin{array}{c}0,44 \mathrm{~b} \\
(11 \%)\end{array}$ & $\begin{array}{c}0,27 \mathrm{~b} \\
(8 \%)\end{array}$ & $\begin{array}{l}0,86 \mathrm{a} \\
(12 \%)\end{array}$ \\
\hline Total & $2,97 \mathrm{c}$ & $2,76 \mathrm{c}$ & $1,37 \mathrm{c}$ & $0,78 \mathrm{c}$ & $3,18 \mathrm{~b}$ & $2,96 \mathrm{~b}$ & $4,06 \mathrm{~b}$ & $3,50 \mathrm{~b}$ & $7,47 \mathrm{a}$ \\
\hline Tratamentos & \multicolumn{2}{|c|}{$\mathrm{T} 1$} & \multicolumn{2}{|c|}{$\mathrm{T} 2$} & \multicolumn{2}{|c|}{$\mathrm{T} 3$} & \multicolumn{2}{|c|}{$\mathrm{T} 4$} & \\
\hline Média mensal & \multicolumn{2}{|c|}{$0,26 \mathrm{~A}$} & \multicolumn{2}{|c|}{$0,24 \mathrm{~A}$} & \multicolumn{2}{|c|}{$0,23 \mathrm{~A}$} & \multicolumn{2}{|c|}{$0,18 \mathrm{~A}$} & \\
\hline
\end{tabular}

, situado em torno de 60 a 80\% da serapilheira total (A RAÚJO et al., 2006; MACHADO et al., 2008).

A fração ramos obteve maior produção na área da floresta secundária, não havendo diferença en tre os tratamentos das unidades de sistemas agroflorestais. Por outro lado, a contribuição da fração parte reprodutiva apresentou deposição irregular entre as áreas de estudo. Tal fato pode estar associado à ocorrência de eventos como florescimento e frutificação das espécies, que podem ocorrer de forma distinta entre as áreas. Quanto à fração resíduos (material oriundo da degradação e ação de organismos e insetos decompositores), foi observada maior produção na área de floresta secundária. Is so pode indicar a maior presença e ação des ses organismos na área de floresta, enquanto entre as unidades de sistemas agroflorestais os valores foram semelhantes e não apresentaram dife renças significativas.

Em relação à produção de serapilheira entre as unidades experimentais dos SAFs, a área do Patrimônio apresentou significativamente maior aporte do que a do Campinho da Independência (Tabela 2). Essa diferença no aporte de serapilheira pode estar relacionada à prática de manejo adotada nas unidades experimentais, uma vez que, na área do Patrimônio, as espécies arbóreas renovadoras de fertilidade (Inga sp., L. guillemineanus, Bixa orellana e C. myriantum) receberam podas periódicas. A poda é uma prática adotada no SAFRA que visa dis ponibilizar bio mass a de forma rápida para a ciclagem de nutrientes (CAMPELLO et al., 2007). Seu emprego pode possibilitar o avanço sucessional da comunidade e aumentar a oferta de matéria orgânica, favorecendo a dinamização da vida no sistema (PENEIREIRO, 1999).

Apesar da diferença entre as áreas, quando comparados os tratamentos de sistemas agroflorestais em relação ao uso de espécies leguminosas (T1 e T2) e não leguminosas (T3 e T4), não foi observada diferença significativa no aporte de serapilheira (Tabela 2). Da mes ma forma, no presente estudo não foi observada diferença significativa no aporte total de serapilheira em relação ao plantio "solteiro" (uma muda/cova) (T1 e T3) e "casado" (duas mudas/cova) (T2 e T4), embora o número de indivíduos no plantio "casado" seja relativamente maior em relação ao plantio "solteiro". Isso se contrapõe aos resultados encontrados por Viera e Schumacher (2010), pois espera-se que o uso de leguminosas arbóreas favoreça a maior produção de serapilheira, devido às suas características de rápido crescimento e ma ior eficiência de utilização dos nutrientes extraídos do solo. Esse resultado possivelmente está relacionado ao comportamento de aporte das espécies de leguminosas utilizadas.

Pelos resultados obtidos, mesmo após cinco anos, os modelos estudados ainda não atingiram valor de aporte de serapilheira similar ao da área de floresta secundária em estádio médio de sucessão $\left(7,47 \mathrm{Mg}^{-} \mathrm{ha}^{-1}\right)$. Contudo, embora esses valores tenham sido inferiores à área de referência, estudos conduzidos em florestas 
secundárias no Rio de Janeiro apresentaram aportes de serapilheira de 2,4 Mg.ha ${ }^{-1}$ (CORRÊA NETO et al., 2001), com maiores valores $\left(4,0 \mathrm{Mg}_{\mathrm{ha}} \mathrm{h}^{-1}\right)$ em áreas mais tardias e menores $\left(2,9 \mathrm{Mg} \mathrm{ha}^{-1}\right)$ nas de estádios mais iniciais (TOLEDO et al., 2002). Por esses dados, apenas a área sem manejo, Campinho da Independência, nos tratamentos sem uso de leguminosas - T3 (1,37 Mg.ha $\left.{ }^{-1}\right)$ e T4 $\left(0,78 \mathrm{Mg} \cdot \mathrm{ha}^{-1}\right)$ - foram verificados valores inferiores aos citados para florestas em sucessão. Portanto, embora aos cinco anos o aporte de serapilheira ainda seja inferior ao de uma floresta em estádio médio de regeneração, os valores obtidos se assemelham aos encontrados em florestas secundárias iniciais.

Em relação à sazonalidade de produção, independentemente da área estudada, a maior produção de serapilheira total foi verificada nos meses de janeiro e agosto (final da estação seca). Porém, entre os tratamentos, os comportamentos foram distintos em relação à sazonalidade dos eventos. No Patrimônio, foram constatados picos na deposição de ramos em novembro, e nas partes reprodutivas, de dezembro a janeiro, enquanto na área de floresta houve dois episódios, sendo um em abril a maio e outro de agos to a setembro (Figura 1).
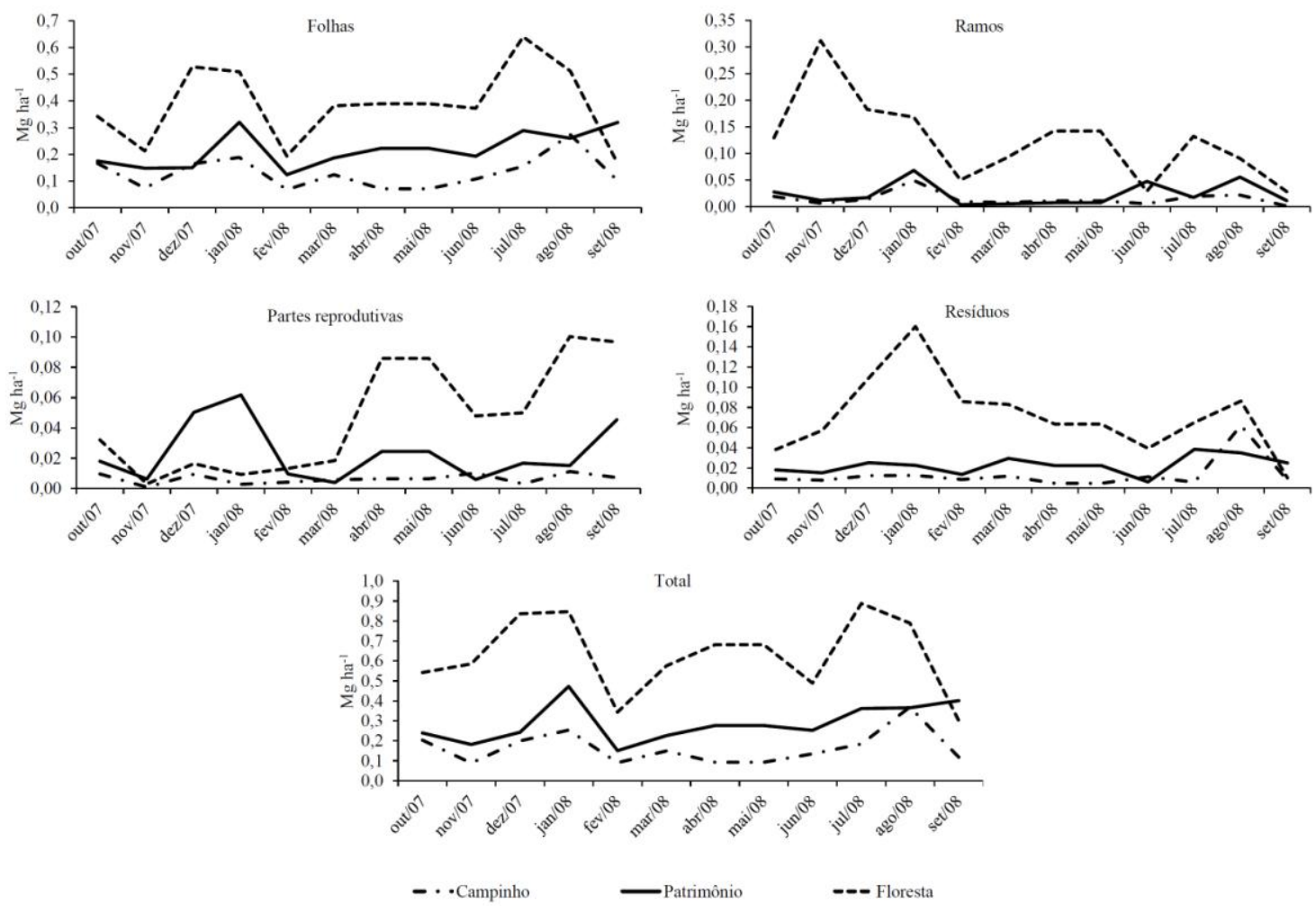

Figura 1. Deposição sazonal das frações de serapilheira nas unidades experimentais de sistemas agroflorestais, Campinho da Independência e Patrimônio, e na área de floresta secundária.

Figure 1. Seasonal deposition of litter fractions in the experimental units of agroforestry systems, Campinho da Independência e Patrimônio, and the area of secondary forest.

Resultados semelhantes foram obtidos por Araújo et al. (2006), em que a maior produção de serapilheira, na estação seca, pode estar relacionada com resposta ao estresse hídrico pelo descarte das folhas (MARTINS; RODRIGUES 1999), principal componente da serapilheira. O comportamento de aporte observado pode ser atribuído à maior diversidade da floresta secundária, com espécies florescendo ao longo de todo o ano, e às práticas de manejo adotadas na área do Patrimônio, com podas e aberturas de copas que favorecem a reprodução de plantas que requerem luz para seu processo reprodutivo.

$\mathrm{O}$ aporte de nutrientes via serapilheira foi significativamente diferente entre os tratamentos estudados $(p<0,01)$. Os maiores valores médios de macronutrientes foram encontrados na floresta secundária, seguindo a tendência do aporte de serapilheira (Tabela 3).

Resultado semelhante foi encontrado por Corrêa et al. (2006), que observou aportes maiores de nutrientes na área de vegetação natural em relação aos sistemas agroflorestais, similar à deposição de biomassa. Segundo Go mes et al. (2010), as variações nas concentrações dos nutrientes podem estar relacionadas à idade da vegetação, à estratificação da floresta e à concentração dos elementos, tanto no solo quanto nas partes da planta. 
Tabela 3. Conteúdos médios de macro e micronutrientes $\left(\mathrm{kg}_{\mathrm{ha}} \mathrm{ha}^{-1} \cdot \mathrm{ano}^{-1}\right)$ nas unidades experimentais de sistemas agroflorestais, aos cinco anos de idade, e na área de floresta secundária, em Paraty, RJ.

Table 3. Average content of macro and micronutrients $\left(\mathrm{kg} \cdot \mathrm{ha}^{-1} \cdot \mathrm{yr}^{-1}\right)$ in experimental units of agroforestry, at five years, and in a secondary forest, Paraty, RJ.

\begin{tabular}{lcccccc}
\hline Local & Tratamento & $\mathbf{N}$ & $\mathbf{P}$ & $\mathbf{K}$ & $\mathbf{C a}$ & $\mathbf{M g}$ \\
\hline \multirow{6}{*}{ Campinho } & T1 & 4,54 & 0,31 & 2,72 & 3,78 & 0,67 \\
& T2 & 3,96 & 0,17 & 1,01 & 3,33 & 0,48 \\
& T3 & 1,69 & 0,13 & 0,69 & 1,63 & 0,29 \\
& T4 & 1,03 & 0,09 & 0,29 & 1,13 & 0,18 \\
& Média & $2,81 \mathrm{~b}$ & $0,18 \mathrm{~b}$ & $1,18 \mathrm{~b}$ & $2,47 \mathrm{c}$ & $0,40 \mathrm{c}$ \\
\multirow{5}{*}{ Patrimônio } & T1 & 4,91 & 0,27 & 1,72 & 4,07 & 0,61 \\
& T2 & 4,93 & 0,22 & 1,06 & 3,62 & 0,53 \\
& T3 & 5,18 & 0,34 & 1,58 & 4,85 & 0,85 \\
Floresta & T4 & 3,8 & 0,27 & 1,08 & 4,91 & 0,83 \\
& Média & $4,70 \mathrm{~b}$ & $0,27 \mathrm{~b}$ & $1,36 \mathrm{~b}$ & $4,36 \mathrm{~b}$ & $0,70 \mathrm{~b}$ \\
& & $16,04 \mathrm{a}$ & $0,80 \mathrm{a}$ & $4,12 \mathrm{a}$ & $11,04 \mathrm{a}$ & $2,34 \mathrm{a}$
\end{tabular}

$\mathrm{T} 1$ = leguminosas com plantio "solteiro"; T2 = leguminosas com plantio "casado";T3 = não leguminosas com plantio "solteiro"; T4 = não leguminosas com plantio "casado".

$\mathrm{O}$ aporte superior de $\mathrm{N}$ na floresta secundária foi numericamente superior aos tratamentos $\mathrm{T} 1 \mathrm{e} \mathrm{T} 2$ das unidades experimentais, onde foram consorciados com espécies leguminosas, as quais têm a capacidade de fixar $\mathrm{N}$ atmosférico. Trabalhos realizados por Fernandes et al. (2006) e Mochiutti et al. (2006) demonstraram que áreas revegetadas com espécies leguminosas apresentaram aporte superior de $\mathrm{N}$ em relação à área de floresta, sendo importantes para a recuperação de solos degradados. Também Silveira et al. (2007) constataram, para vários modelos de SAFRA na região de Paraty, aporte de $\mathrm{N}$ de 1,4 a 2,6 vezes superior na área de floresta em estágio inicial de sucessão. Corrêa (2006), estudando a ciclagem de nutrientes em diferentes coberturas frutíferas e florestais de um sistema agroflorestal multiestratificado, não encontrou diferença de $\mathrm{N}$ entre os componentes dos SAFs, assim como este estudo, mas observou uma leve superioridade na concentração desse nutriente na serapilheira da vegetação natural. Nesse mesmo estudo, o autor observou diferença entre a concentração de nutrientes das folhas das árvores e da serapilheira depositada, concluindo que as espécies reabsorvem o nutriente das folhas como alternativa estratégica para a conservação do nutriente pelas plantas.

Dess a forma, pode-se considerar a hipótese de que houve um potencial efeito da composição do SAFRA estudado, que pode ter afetado os teores de nutrientes presentes no sistema. Es se resultado pode refletir o efeito da densidade de plantio de Bactris gasipae, a qual é considerada uma espécie que demanda alta dosagem de $\mathrm{N}$ para seu desenvolvimento em solos pobres (BOVI et al., 2002). O Campinho da Independência também apresentou menores valores nas médias dos nutrientes $\mathrm{Ca}$ e $\mathrm{Mg}$, sendo estes significativamente diferentes dos valores obtidos na área do Patrimônio. Ess a diferença no aporte de nutrientes pode estar relacionada à prática de manejo adotad a na área do Patrimônio, que possibilitou a mesma resposta do sistema, independentemente da presença ou ausência de leguminosas. Ao comparar o aporte de nutrientes na serapilheira de um SAF orientado pela sucessão natural ao de uma área de capoeira de mesma idade, Peneireiro (1999) concluiu que o manejo da poda da vegetação foi o maior responsável pelas diferenças entres as duas áreas estudadas, conduzindo a área manejada a uma condição sucessional mais avançada, com maior oferta de matéria orgânica e concentração de nutrientes.

Ao analisar o comportamento do aporte de serapilheira como indicador ambiental para recuperação de APPs, foi possível observar a sua sensibilidade e efetividade, permitindo identificar comportamentos distintos entre as áreas estudadas, indicando sua capacidade de refletir alterações do meio e das práticas de manejo adotadas nas unidades experimentais. Em outros trabalhos realizados, a serapilheira também foi considerada como um indicador eficiente na restauração de áreas degradadas (MARTINS; RODRIGUES, 1999; ARAÚJO et al., 2006; MACHADO et al., 2008).

\section{CONCLUSÕES}

- A não equiparação do Sistema Agroflorestal Regenerativo e Análogo (SAFRA) com uma flore sta secundária de cinco anos indica a necessidade de um acompanhamento de longo prazo, para avaliar sua funcionalidade ecológica de restauração florestal. Por outro lado, ficou evidenciada a viabilidade desse sistema quando associado a práticas de manejo da vegetação e poda. Nessas condições, até aos cinco, mostrou-se eficiente para restaurar o aporte de biomassa ao solo. Contudo, para a aplicação desses modelos é necessária a análise das espécies e sua densidade de plantio, devido à redução constatada nos conteúdos de nutrientes, em especial N, quando comparados a outros modelos de SAFs biodiversos e de florestas em 
diferentes estádios sucessionais. Com isso, o aporte de serapilheira pode ser considerado um eficiente indicador para monitorar a funcionalidade ecológica de sistemas agroflorestais .

\section{REFERÊNCIAS}

ALVARES, C. A.; STAPE, J. L.; SENTElHAS, P. C.; GONÇALVES, J. L. M.; SPAROVEK, G. Köppen's climate classification map for Brazil. Meteorologische Zeitschrift, Stuttgart, v. 22, n. 6, p. 711 - 728, 2013.

ARAÚJO, R. S.; PIÑA-RODRIGUES, F. C. M.; MACHADO, M. R.; PEREIRA, M. G.; FRAZÃO, F. J. Aporte de serrapilheira e nutrientes ao solo em três modelos de revegetação na Reserva Biológica de Poços das Antas, Silva Jardim, RJ. Floresta e Ambiente, Seropédica, v. 12, n. 2, p. 15 - 21, 2006.

ASHFORD, O. S.; FOSTER, W. A.; TURNER, B. L.; SA YER, E. J.; SUTCLIFFE, L.; TANNER, E. V. J. Litter manipulation and the soil arthropod community in a lowland tropical rainforest. Soil Biology \& Biochemistry, Oxford, v. 62, p. 5 - 12, 2013.

BOVI, M. L. A.; GODOY JÚNIOR, G.; SPIERING, S. H. Respostas de crescimento da pupunheira à adubação NPK. Scientia Agricola, Piracicaba, v. 59, n. 1, p. 161 - 166, 2002.

CAMPELlO, E. F. C.; SILVA, G. T. A.; NÓBREGA, P. O.; VIEIRA, A. L. M.; FRANCO, A. A.; RESENDE, A. S. Sistemas agroflorestais na Mata Atlântica: a experiência da Embrapa Agrobiologia. Seropédica: Embrapa Agrobiologia (Circu lar Técnica 21), 2007. 7 p.

CONSELHO NACIONA L DO MEIO AMBIENTE (CONAMA). Resolução CONAMA 01, de 31 de janeiro de 1994. Regulamenta o art. $6^{\circ}$ do Decreto no 750, de 10 de fevereiro de 1993 para o Estado de São Paulo. Ministério do Meio Ambiente, Brasília, DF, 31 jan. 1994. Disponível em: <http://www.sigam.ambiente. sp.gov.br/Sigam2/repositorio/222/documentos/RES_CONAMA_1994_001.pdf>. Acesso em: 20 dez. 2013.

CORRÊA NETO, T. A.; PEREIRA, M. G.; CORREA, M. E. F.; ANJOS, L. H. C. Deposição de serrapilheira e mesofauna edáfica em áreas de eucalipto e floresta sucessão secundária espontânea. Floresta e Ambiente, Seropédica, v. 8, n. 1, p. 70 - 75, 2001.

CORRÊA, F. L. O.; RAMOS, J. D.; GAMA-RODRIGUES, A. C.; MULLER, M. W. Produção de serapilheira em sistema agroflorestal multiestratificado no estado de Rondônia, Brasil. Ciência e Agrotecnologia, Lav ras, v. 30, n. 6, p. $1099-1105,2006$.

CUNHA NETO, F. V.; LELES, P. S. S.; PEREIRA, M. G.; BELLUMATH, V. G. H.; ALONSO, J. M. Acú mulo e decomposição da serapilheira em quatro formações florestais. Ciência Florestal, Santa Maria, v. 23, n. 3, p. $379-387,2013$

ESPIG, S. A.; FREIRE, F. J.; MARANGON, L. C.; FERREIRA, R. L. C.; FREIRE, M. B. G. S.; ESPIG, D. B. Sazonalidade, composição e aporte de nutrientes da serapilheira em frag mento de Mata Atlântica. Revista Árvore, Viçosa, v. 33, n. 5, p. 949 - 956, 2009.

FERNANDES, M. M.; PEREIRA, M. G.; MA GALHÃES, L. M. S.; CRUZ, A. R.; GIÁCOMO, R. G. Aporte e decomposição de serapilheira em áreas de floresta secundária, plantio de sabiá (Mimosa caesalpiniaefolia Benth.) e andiroba (Carapa guianensis Aubl.) na flona Mário Xavier, RJ. Ciência Florestal, Santa Maria, v. 16, n. 2, p. $163-175,2006$.

GOMES, J. M.; PEREIRA, M. G.; PIÑA-RODRIGUES, F. C. M.; PEREIRA, G. H. A.; GONDIM, F. R.; SILVA, E. M. R. Aporte de serapilheira e de nutrientes em frag mentos florestais da Mata Atlântica, RJ. Revista Brasileira de Ciências Agrárias, Recife, v. 5, n. 3, p. 383 - 391, 2010.

INSTITUTO BRASILEIRO DE GEOGRAFIA E ESTATÍSTICA (IBGE). Manual técnico da vegetação brasileira. 2. ed. Rio de Janeiro: IBGE. 2012. 271 p.

MACHADO, M. R.; PIÑA-RODRIGUES, F. C. M.; PEREIRA, M. G. Produção de serapilheira como bioindicador de recuperação em plantio adensado de revegetação. Revista Árvore, Viçosa, v. 32, n. 1, p. 143 151,2008

MARTINS, S. V.; RODRIGUES, R. R. Produção de serrapilheira em clareiras de uma floresta estacional semidecidual no município de Campinas, SP. Revista Brasileira de Botânica, São Paulo, v. 22, n. 3, p. 405 412, 1999.

FLOREST A, Curitiba, PR, v. 46, n. 1, p. 75 - 82, jan. / mar. 2016

Souza, M. C. S. de et al.

ISSN eletrônico 1982-4688

DOI: $10.5380 /$ rf.v46il .34991 
MOCHIUTTI, S.; QUEIROZ, J. A. L.; MELÉM JUNIOR, N. J. Produção de serapilheira e retorno de nutrientes de um povoamento de taxi-branco e de u ma Floresta Secundária no Amapá. Boletim de Pes quisas Florestais, Colo mbo, n. 52, p. 3 - 20, 2006.

NAEEM, S. Biodiversity and ecosystem functioning in restored ecosystems: extracting principles for a synthetic perspective. In: FALK, D. A.; PALMER, M. A.; ZEDLER, J. B. (Eds.). Foundation of restoration ecology. Washington: Island Press, 2006. p. 210 - 237.

NASCIMENTO, A. F. J.; SILVA, T. O.; SAMPAIO, E. V. S. B.; ARAÚJO FILHO, R. N.; DANTAS, T. V. P. Quantificação de serapilheira em diferentes áreas sob fragmentos do Parque Nacional Serra de Itabaiana, Sergipe. Semina: Ciências Agrárias, Londrina, v. 34, n. 6, p. 3271 - 3284, 2013.

OLIVEIRA, R. E.; ENGEL, V. L. A restauração ecológica em destaque: um retrato dos últimos vinte e oito anos de publicações na área. Oecologia Australis, Rio de Janeiro, v. 15, n. 2, p. 303 - 315, 2011.

PENEIREIRO, F. M. Sistemas agroflorestais dirigidos pela sucessão natural: um estudo de caso. $149 \mathrm{f}$. Dissertação (Mestrado. Ciências Florestais) - Escola Superior de Agricultura "Luiz de Queiroz", Piracicaba, 1999.

RAMOS-FILHO, L. O; FRANCISCO, C. E. S.; ALY JUNIOR, O. Legislação ambiental e uso de sistemas agroflorestais em assentamentos rurais no estado de São Paulo. Revista Brasileira de Agroecologia, São Paulo, v. 2, n. 1, p. $280-283,2007$.

SILVEIRA, N. D.; PEREIRA, M. G.; POLIDORO, J. C.; TAVARES, S. R. L.; MELlO, R. B. Aporte de nutrientes e biomassa via serrapilheira em sistemas agroflorestais em Paraty, RJ. Ciência Florestal, Santa Maria, v. 17, n. 2, p. 129 - 136, 2007.

TOLEDO, L. O.; PEREIRA, M. G.; MENEZES, C. E. G. Produção de serrapilheira e transferência de nutrientes em florestas secundárias localizadas na região de Pinheiral, RJ. Ciência Florestal, Santa Maria, v. 12, n. 2, p. 9 16, 2002.

VIERA, M.; SCHUMACHER, M. V. Deposição de serapilheira e de macronutrientes em um povoamento de acácia-negra (Acacia mearnsii De Wild.) no Rio Grande do Sul. Ciência Florestal, Santa Maria, v. 20, n. 2, p. $225-233,2010$.

VIVAN, J.; FLORIANI, G. S. Construção participativa de indicadores de sustentabilidade em sistemas agroflorestais em rede na Mata Atlântica. In: VILCAHUAMÁN, L. J. M.; RIBASKI, J.; MACHADO, A. M. B. (Org.). Sistemas Agroflorestais e desenvol vimento com proteção ambiental: práticas e tecnologias desenvolvidas. Curitiba: Embrapa Florestas, 2006. 214 p. 